\title{
Early Vascular Ageing - A Concept in Development
}

\author{
Peter M Nilsson
}

Professor, Lund University, Malmö, Sweden

\begin{abstract}
Cardiovascular disease (CVD) is a prevalent condition in the elderly, often associated with metabolic disturbance and type 2 diabetes. For a number of years, research dedicated to understand atherosclerosis dominated, and for many good reasons, this pathophysiological process being proximal to the CVD events. In recent years, research has been devoted to an earlier stage of vascular pathology named arteriosclerosis (arterial stiffness) and the new concept of early vascular ageing (EVA), developed by a group of mostly European researchers. This overview describes recent developments in research dedicated to EVA and new emerging aspects found in studies of families at high cardiovascular risk. There are new aspects related to genetics, telomere biology and the role of gut microbiota. However, there is still no unifying definition available of EVA and no direct treatment, but rather only recommendations for conventional cardiovascular risk factor control. New interventions are being developed - not only new antihypertensive drugs, but also new drugs for vascular protection - the selective angiotensin-II (AT2) agonist Compound 21 (C21). Human studies are eagerly awaited. Even new functional food products could have the potential to positively influence cardiometabolic regulation, to be confirmed.
\end{abstract}

\section{Keywords}

Arterial ageing, arterial stiffness, blood pressure, C21, diet, drugs, glycaemia, hypertension, lipids, microbiota

Disclosure: Peter M Nilsson has no conflicts of interest to declare. No funding was received for the publication of this article. This review was supported by two grants from the Research Council of Sweden for research on vascular ageing and the family clustering of cardiometabolic disorders.

Open Access: This article is published under the Creative Commons Attribution Noncommercial License, which permits any non-commercial use, distribution, adaptation and reproduction provided the original author(s) and source are given appropriate credit.

Received: 4 February 2015 Accepted: 13 March 2015 Citation: European Endocrinology, 2015;11(1):26-31 DOI:10.17925/EE.2015.11.01.26

Correspondence: Peter M Nilsson, Lund University, Department of Clinical Sciences, Skåne University Hospital, IM Nilssons gata 32 , S-205 02 Malmö, Sweden.

E: Peter.Nilsson@med.lu.se

Thomas Sydenham, the great English seventeenth-century physician (see Figure 1), said that "A man is as old as his arteries", a concept that has once again come into focus of cardiovascular research and clinical activities, which this review aims to describe.

In preventive cardiology there has long existed the need to target individuals at risk for preventive action based on lifestyle improvement and intervention related to conventional risk factors for cardiovascular disease (CVD) manifestations (hyperlipidaemia, hypertension, smoking, diabetes). This has been made possible by use of specific risk algorithms based on follow-up investigations of screened subjects for prediction of events in large population-based cohorts. Famous examples include the Framingham Risk Score (FRS) ${ }^{1}$ and the European SCORE, which originated with the Danish algorithm PRECARD, ${ }^{2}$ as well as other lessknown algorithms (PROCAM from Germany; Q-RISK from the UK). The SCORE system has been calibrated to national applications based on national cardiovascular statistics on CVD endpoints. Computer applications have been widely distributed, even when the clinical uptake is difficult to quantify.

At least the US-based FRS has been found to overestimate the cardiovascular risk in many European populations, providing a reason for using SCORE instead. ${ }^{3,4}$ Furthermore, new versions of SCORE, including high-density lipoprotein (HDL) cholesterol and not only total cholesterol, will reclassify patients more correctly for risk categories than does the traditional SCORE. ${ }^{5}$ For cardiovascular complications of type 2 diabetes, some other risk algorithms have been developed in newly detected patients, based mostly on the UK Prospective Diabetes Study (UKPDS) Risk Engine, ${ }^{6}$ but also based on national register data on treated patients with type 2 diabetes from Sweden. ${ }^{7}$ The endpoint most commonly used for these algorithms was cardiovascular events (fatal or non-fatal), generally caused by atherosclerosis, plaque rupture or thrombo-embolic mechanisms. In more recent years, the research activities dedicated to atherosclerosis also started to involve effects of acute or chronic inflammation mediating the risk of CVD - for example, in patients with the metabolic syndrome. ${ }^{8}$ Inflammation is often linked to abdominal obesity, insulin resistance and hepatic steathosis and supposed to be both general and local - that is, perivascular by origin. The modern genetic studies and discoveries of CVD and type 2 diabetes have contributed to our understanding of causal pathways for these disorders, as revealed by applying so-called causal inference (Mendelian randomisation) methodologies, ${ }^{9}$ but so far have not resulted in new drug developments. Even if substantial achievements have been accomplished both in pathophysiology and evidence-based treatment, there is need for deeper understanding of the early origins and features of cardiovascular and metabolic disease, as well as elucidation of why many of these disorders tend to cluster in families at risk not directly addressed in the conventional risk algorithms. This is a background for the emerging interest dedicated to arterial stiffness (arteriosclerosis) and the development of the concept of early vascular ageing (EVA) since 2008. It is noteworthy that the previous concept of the metabolic syndrome has been heavily criticised since 2005, mostly by diabetologists, for inherent problems with definitions and applications in daily practice, but also for the lack of specific treatment. ${ }^{10}$ Thus the arena was open to 
new concepts in cardiometabolic medicine to support understanding of pathophysiology and prevention. Such new concepts will one day also challenge the EVA construct and are a reflection of how science works in a critical way.

\section{Arterial Stiffness as a Reflection of Vascular Ageing}

Attempts have been made to define arterial ageing during the development from normal ageing of arteries to disease caused by superimposed atherosclerosis. ${ }^{11}$ Many researchers now consider arterial stiffness (arteriosclerosis) to represent an earlier step in vascular pathology during the life course than atherosclerosis, as arterial stiffness could possibly be influenced to a high degree by factors acting already in early life. Atherosclerosis leading to plaque formation and rupture, causing clinical disease events, is better defined and has been associated with a number of well-known risk markers or risk conditions. ${ }^{12}$ Among these are genetic factors, especially involving lipid metabolism, as well as adverse lifestyle factors (smoking, sedentary lifestyle, unhealthy diet) and traditional risk factors such as hypertension and diabetes, but also less-well-defined factors such as chronic inflammation and the influence of oxidative stress.

As every human being has once been pre-formed in utero, some hypotheses hold that early life influences could play a role in development of arterial function and vascular morphology, leading to increased risk of arteriosclerosis as visible at least in young adulthood. All components of the vascular tree may be affected - for example, the elastin content and thinness of the arterial wall; the development of the microcirculation, as can be studied in the retinal bed; and, finally, the number and functionality of capillaries.

The famous mismatch hypothesis denotes the detrimental effects of intrauterine growth retardation (IUGR) in combination with post-natal catch-up growth patterns for increased risk of CVD and type 2 diabetes in adulthood. ${ }^{13}$ Because these changes are also related to ageing, it has been proposed that a process of EVA is an early sign of arteriosclerosis (in the media) but is linked also to early changes in the endothelial function (intima), to haemodynamic changes and to the influence of abnormal glucose metabolism and increased inflammation, as reviewed. ${ }^{14,15}$ The links with type 2 diabetes are shown in Table 1 The difference between the concept of arterial ageing and EVA is that the latter also encompasses changes in the smaller arteriolae and the microcirculation based on the cross-talk between the macro- and micro-circulation as evident in the origin of vascular brain damage. ${ }^{16}$ EVA is now being extensively studied in different population-based cohorts, in Europe, Asia and Latin America, but still no general definition has been agreed upon. One proposed way to define EVA could be to use the outliers according to the normal range of carotid-femoral pulse wave velocity (C-f PWV) - that is, above the 2 standard deviations (SDS) of the normal distribution of $\mathrm{c}-\mathrm{f}$ PWV based on data from the European reference group..$^{17}$ Another way to describe EVA is based on statistical methods when arterial stiffness ( $c-f$ PWV), a central aspect of EVA, is used as the dependent variable in multiple regression analyses and a number of risk markers are used as independent variables based on data from population-based studies. Because the influence of haemodynamic changes and sympathetic nervous system (SNS) stimulation on the arterial tone is substantial, the data are normally adjusted for mean arterial pressure (MAP) and heart rate (HR), the latter being a marker of SNS activity, especially relevant if resting conditions when arterial stiffness is measured are not well standardised.

\section{Figure 1: Thomas Sydenham, 'The English Hippocrates', 1624-1689}

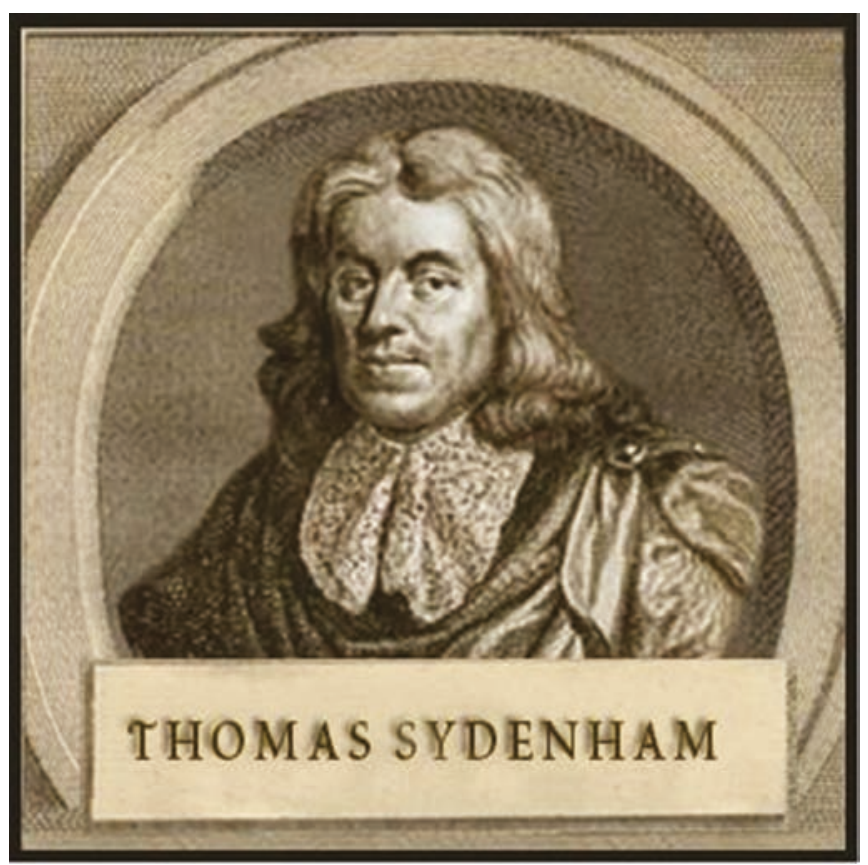

Such investigations in a population-based study of elderly subjects from the Malmö Diet Cancer (MDC) Study cardiovascular cohort $(n=3,700$, mean age 71$)$ in Malmö, Sweden, has revealed that markers of glucose metabolism and dyslipidaemia (elevated triglycerides, low HDL-cholesterol levels), as well as increased waist circumference (a marker of active abdominal fat tissue with inflammatory action), are significantly associated with arterial stiffness (c-f PWV) but not lowdensity lipoprotein (LDL)-cholesterol, smoking or cystatin-C, a marker of impaired renal function. ${ }^{18}$ Our own interpretation is thus that two different clusters of CVD risk factors could be shown and that these are, relatively speaking, differently involved along the life course for development, first, of arteriosclerosis and, later on, of atherosclerosis, starting in young adulthood and mid-life. Finally, in elderly subjects, there may exist a merged phenotype of combined arterio- and atherosclerosis close to clinical events and symptomatic coronary heart disease or CVD, as well as peripheral artery disease.

In the MDC study, levels of arterial stiffness were also higher in subjects having known or newly detected diabetes compared with subjects without diabetes (see Figure 2). In another set of analyses from the same cohort, it was shown that arterial stiffness (c-f PWV), at least at the higher end of the distribution, is associated with impaired cognitive function as measured in a cross-sectional analysis, especially concerning so-called speed and executive testing, but not with traditional memory tests such as Mini-Mental State Examination (MMSE). ${ }^{19}$ Vascular ageing thus links to brain and cognitive ageing.

\section{Genetic Factors Associated with Arterial Stiffness}

Attempts have been made to think about how to define EVA by taking into account background factors such as age, sex and ethnicity, but what can we learn from genetics? There is now a list of about 40 genetic markers of hypertension ${ }^{20}$ that in a joint Italian-American study did not seem to overlap with other genetic markers associated with arterial stiffness. ${ }^{21}$ Still more work has to be done to understand the genetic 


\section{Table 1: Links between Type 2 Diabetes and the Early Vascular Ageing Syndrome}

\author{
Arterial stiffness \\ Haemodynamic ageing, hypertension \\ Microvascular and endothelial dysfunction \\ Chronic inflammation, general and perivascular \\ Insulin resistance \\ Defects in incretin function \\ Hyperglycaemia, AGE \\ Dyslipidaemia \\ Early life influences \\ Telomere shortening/attrition \\ Cognitive dysfunction and brain ageing \\ Dysbiosis of the gut microbiota \\ Arterial media calcification in nephropathy \\ Neuropathy and autonomous nerve dysfunction \\ $A G E=$ advanced glycation end products .
}

map of arterial stiffness and the differences towards other conditions, such as atherosclerosis, on a genetic basis. Such new genetic studies on arterial stiffness are published, ${ }^{22}$ but ideally more population-based cohorts should contribute data for a stronger statistical power to elucidate on these markers. If the genes can be defined, normally the gene products can also be. There are, for example, hints that genetic markers of collagen synthesis are linked to arterial stiffness. Ideally, this could translate into finding new drug targets for a more causally directed intervention strategy against arterial stiffness, to be complemented by conventional drug therapy for hypertension and hyperlipidaemia.

\section{Telomere Length and Vascular Ageing - A Methodological Challenge}

The role of telomere length in relation to arterial stiffness and vascular ageing is still not well understood, and even if telomere length is regarded as a marker of biological ageing, it has not been uniformly associated with vascular ageing - for example, in the Belgian Asklepios study based on cross-sectional data in largely healthy individuals. ${ }^{23}$ The methodological solution to this could be to use repeated measurements of telomere length during follow-up to calculate the so-called telomere attrition rate, which could be a better marker of ageing than cross-sectional measures alone. In addition, the analyses of other aspects of telomere biology, such as telomerase function and telomere binding proteins, could contribute to deeper understanding than telomere length itself.

\section{Prediction of Cardiovascular Disease Based on Arterial Stiffness}

Increased c-f PWV as a marker of arterial stiffness is an independent risk marker for cardiovascular events and total mortality, even adjusted for traditional risk factors, and even more so in the middle-aged compared with the elderly. ${ }^{24}$ This age-linked influence could mean that differential ageing in general, and vascular ageing in particular, is more visible in middle-aged subjects than in the elderly, among whom also selective survival bias could play a role. Two consensus papers have been published: one from 2006 on methods to evaluate $\mathrm{c}-\mathrm{f} \mathrm{PWV} ;{ }^{25}$ one from 2012 on a defined risk threshold of $10 \mathrm{~m} / \mathrm{s}$ for $\mathrm{c}-\mathrm{f} \mathrm{PWV}{ }^{26}$ reflecting arterial stiffness and a recommendation for standardisation of measurement. The statement is that the crucial length component should be calculated by direct measurement of the distance between the carotid and the femoral arteries but multi-plicated with $0.8 .{ }^{26}$ The travelled distance of the pulse wave may be shorter than the one calculated by external tapes, especially if a profound abdominal obesity exists. Preferably, in such patients, a special modifiable caliper, so called, should be used to avoid bias from the protruding abdominal volume.

Studies have shown how closely correlated arterial stiffness is with age and hypertension, as well as some other risk conditions. ${ }^{27,28}$ In the Baltimore Longitudinal Study of Ageing (BLSA), it has been reported that arterial stiffness can even predict the development of hypertension ${ }^{29}-$ another strong argument for early prevention and intervention with the ambition to prevent hypertension and thereby target organ damage at an early stage. The same prediction of hypertension was seen in the Framingham cohort. ${ }^{30}$

Another important factor is impaired glucose metabolism and hyperglycaemia with effect on the properties of the arterial wall arising from glycation of vessel wall proteins, but also changes of other functions, such as innervation, vascularisation of the arterial wall (via vasa vasorum) and changes in the perivascular fat with inflammation. In pre-diabetes or subjects who have the metabolic syndrome, as well as in established diabetes, these changes can be seen, as described. ${ }^{31}$ Prenner et al. even state that some data suggest that arterial stiffness can predict the onset of diabetes. ${ }^{32}$ However, more research is needed to understand whether large artery stiffness and the pulsatile haemodynamic changes that accompany it are involved in the pathogenesis of diabetes. An even more intriguing question is how interventions targeting arterial stiffness could be used to improve clinical outcomes in diabetes.

A specific feature of the arterial wall in patients who have end-stage renal disease (ESRD) is vascular calcification of the media. This is different from conventional atherosclerosis of the intima but can contribute to aortic stiffness. ${ }^{33}$ It is still an open question whether this process starts earlier in the course of chronic kidney disease (CKD) - in fact so early that conventional imaging will not be able to detect it, but rather only specific imaging for metabolic processes. There is no doubt that a close link exists between CKD and CVD as well as with metabolic changes for example, secondary hypercholesterolaemia - or that the degree of albuminuria is an easy-to-quantify marker of this increased risk.

\section{Obesity and Arterial Stiffness}

It is well known that obese children and adolescents, even young adults, tend to have a phenotype of hyperkinetic circulation and vasodilation - the opposite to arterial stiffness - but also a relative endothelial dysfunction. This could lead to increasing blood pressure levels, remodelling of small arteries and, later on, an increase in peripheral vascular resistance and blood pressure, leading to overt hypertension when arterial stiffness is also a gradually increasing feature. The exact regulation of the transition from relative vasodilation in the young obese subject to arterial stiffness in adults is not known, but genetic factors, arterial structure, endothelial dysfunction with impaired nitric oxide (NO) production and the time factor itself could contribute to these changes.

Results from a prospective study showed that central obesity predicts arterial stiffness over a period of 16 years, ${ }^{34}$ whereas a follow-up study 20 years later, including men, indicates that heavy smoking, C-reactive protein (CRP) and pulse pressure (PP) are predictors of arterial stiffness. These data reinforce the importance of chronic inflammation for the development of EVA, as already discussed. However, no study data are available to show cardiovascular benefits by specific target or to treat chronic inflammation. On the other hand, the use of non-steroidal anti-inflammatory agents (NSAIDS) has been associated with increased 
CVD risk, in particular congestive heart failure. Thus the role of chronic inflammation as a target for the treatment strategy is still elusive.

\section{Gene-Environment Interaction in Cardiometabolic Risk}

The family background of many chronic disease conditions is well known and is often asked after during the clinical consultation. There are examples of families at high cardiometabolic risk, and in one register-based study, a special mother-son CVD risk association was described. ${ }^{35}$ The family connection has been documented in several epidemiological studies and also brought into focus based on genetic studies. One conceptual problem to understand is the limited power of combined genetic risk scores (GRS) to explain more than a tiny proportion of this family-associated disease risk. This is why researchers have now tried to define the so-called missing heritability (non-GRS-mediated) believed to involve the influence of gene-environment interactions (epigenetics), lifestyle, early life programming and even the role played by gut microbiota, with its high degree of family resemblance. ${ }^{36}$ In fact, the role of microbiota for risk of cardiometabolic disease is emerging, and even if links have been shown with atherosclerosis, ${ }^{37}$ still no studies have reported on the association between gut microbiota patterns and arterial stiffness. Such analyses are ongoing in the Malmö Offspring Study (MOS) in the city of Malmö, Sweden. In the US, a new study tries to examine the role of gut microbiota for resistant hypertension, a condition with increased arterial stiffness and CVD risk (www.clinicaltrials.gov; ClinicalTrials. gov identifier: NCT02188381). In that ongoing study, a treatment arm together with tetracycline therapy is included to change microbiota patterns and follow effects on vascular regulation and blood pressure levels. Another way of potential treatment is to develop functional food products for supporting health patterns of the gut microbiota-for example, by different Lactobacillus strains in yogurt and dairy products.

\section{Specific Treatment of Arterial Stiffness}

Different methods have been proposed for treating or retarding the process of arterial stiffness as a reflection of vascular ageing. Thus far, it has been shown that a prolonged control of hypertension will reverse early vascular changes and have a long-term beneficial influence on arterial stiffness with decreasing c-f PWV levels over time, beyond the blood pressure control itself. ${ }^{38}$ However, an ongoing randomised controlled study in France, the Statégie de Prévention Cardiovasculaire Basée sur la Rigidité Arterielle Study (SPARTE) aims to compare a treatment strategy for reduction of arterial stiffness (c-f PWV) by different means, including drugs that specifically influence the renin-angiotensin system (RAS) versus another treatment strategy (control) to go for implementation of controlling conventional risk factors including blood pressure, as suggested in European guidelines. ${ }^{39}$ SPARTE is supposed to continue for a number more years yet until a sufficient number of cardiovascular endpoints accumulate to show potential differences in outcomes between treatment arms. Recruitment is ongoing, and eventually also for patients outside France in collaboration with the European Society of Hypertension (ESH).

Blockade of the RAS is supposed to be beneficial for reduction of arterial stiffness beyond blood pressure control per se. There are few data regarding the role of aliskiren, a direct renin inhibitor (DRI), on the central haemodynamics and endothelial function in patients who have uncontrolled arterial hypertension. One study assessed the addition of aliskiren to other antihypertensive drug treatment for arterial stiffness and endothelial function. ${ }^{40}$ Thirty uncontrolled hypertensive patients

\section{Figure 2: Level of Arterial Stiffness (c-f PWV) in Subjects with and without Diabetes}

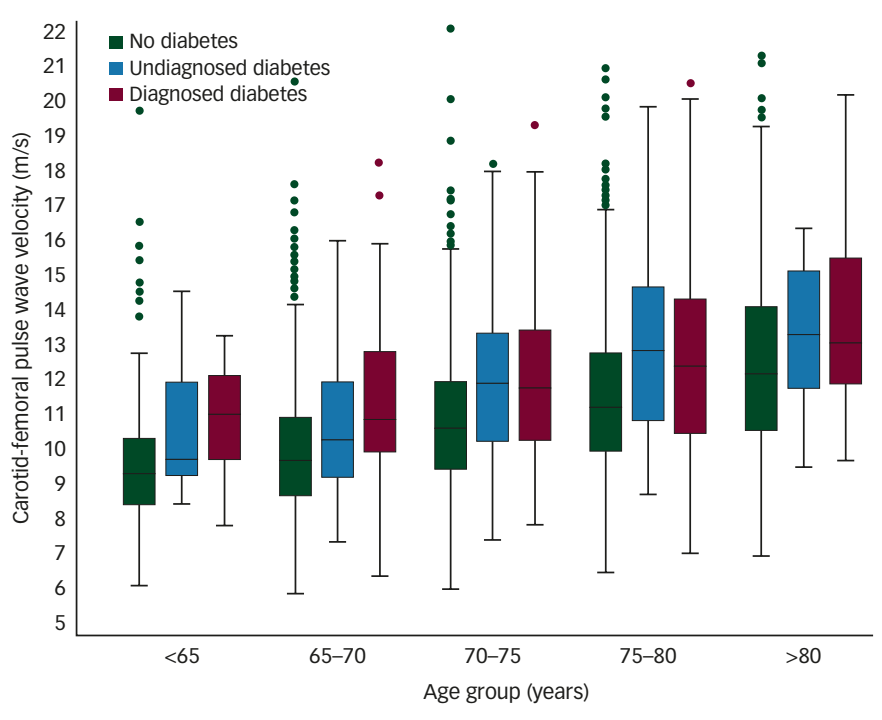

No diabetes: green bar; 83 \%; undiagnosed diabetes: blue bar; 7 \%; or diagnosed, prevalent diabetes: red bar; 10 \% in the Malmö Diet Cancer Study, Malmö, sweden (adapted from reference 18).

(mean age 60.4) not having any other cardiovascular risk factors were enrolled. Augmentation index (AIX) and c-f PWV were measured by applanation tonometry at baseline and after 6 months of aliskiren titrated to $300 \mathrm{mg}$ once a day. The addition of aliskiren had no effect on values of central Alx but significantly improved values of c- $\mathrm{PWV}(9.4 \mathrm{~m} / \mathrm{s}$ versus $8.7 \mathrm{~m} / \mathrm{s} ; \mathrm{p}=0.04)$. In addition to improving systolic and diastolic blood pressure, the addition of aliskiren to concomitant antihypertensive drugs in uncontrolled hypertensive patients may thus be effective in improving aortic stiffness and endothelial function. However, endpoint studies are needed to prove the overall benefits as previously the ALTITUDE study could not show added cardiovascular benefits in patients with type 2 diabetes. ${ }^{41}$ Other groups of patients with diabetes should be evaluated after RAS blockade using different agents, for which many new drugs are currently being developed, as reviewed by Laurent et al. in The Lancet. ${ }^{42}$

Another new approach, focusing on vascular calcifications, has opened up the therapeutic field for new interventions. Substantial and relevant clinical and basic science evidence suggests that modulating the receptor activator for nuclear factor $\kappa B$ (RANK), the RANK ligand (RANKL) and osteoprotegerin (OPG) - that is, RANKL-RANK-OPG signalling, but also the receptor for advanced glycation end products (RAGE) signalling and the associated pro-inflammatory milieu - alters the natural course of cardiovascular complications and outcomes in people who have overt diabetes. ${ }^{43}$ Vascular calcification of the arterial media is also a hallmark for changes seen in the abdominal aorta in patients who have CKD. ${ }^{44}$ These new treatment alternatives must be further tested in controlled clinical studies in patients with various classes of CKD and cardiovascular co-morbidities.

\section{Multiple Cardiovascular Risk Factor Control for Early Vascular Ageing}

As increased c-f PWV has been documented as an independent risk marker for future cardiovascular events and total mortality in a recent meta-analysis, ${ }^{24}$ there is a need to target it with multiple risk factor control, aiming for c-f PWV $<10 \mathrm{~m} / \mathrm{s}$ that is the current threshold for increased risk. ${ }^{26}$ Still, however, no specific treatments exist to target arterial stiffness itself, and no randomised trial is known to have 


\section{Cardiovascular Risk}

investigated this except for a few published studies based on dietary interventions, such as plant stanol ${ }^{45}$ and tart cherry juice, ${ }^{46}$ with varying effects. A randomised, controlled trial for lowering arterial stiffness is thus much needed. At www.clinicaltrials.gov, a few intervention studies targeting arterial stiffness are listed, but no publication seems to be available at present.

Whether control of arterial stiffness would be beneficial for patients who have established type 2 diabetes is currently not known, but it is plausible that it will take a multidrug intervention to achieve positive results in these patients because of the advanced stage of vascular disease and the combination of arteriosclerosis, atherosclerosis and chronic inflammation, further enhanced by hyperglycaemia, insulin resistance and hyperglycaemia. This strategy aiming for cardiovascular prevention in general is also emphasised in the recent European guidelines 2013 on risk factor control in patients who have diabetes, pre-diabetes or impaired glucose metabolism. ${ }^{47}$ In clinical practice, this means that cardiovascular risk patients should be offered antihypertensive and lipidlowering drugs (statins) as well as anti-diabetes drugs if needed, but also smoking cessation (facilitated by drugs if necessary) and aspirin, though the latter only in secondary prevention. ${ }^{47}$

\section{New and Emerging Therapies}

Recent new discoveries providing better understanding of the RAS have led to the development of a selective angiotensin-II (AT2) receptor agonist called compound 21 (C21). This relatively small molecule, discovered 20 years ago in Uppsala, Sweden, increases physiological defence mechanism in the vascular system, providing some vascular protection based on anti-inflammation and anti-remodelling of the arterial wall. Although only data from animal experiments are yet available, ${ }^{48}$ these have shown a reduction of arterial stiffness in experimental models of induced hypertension. ${ }^{49}$ Interestingly, the blood pressure effect by C21 is rather limited why this treatment is a way to selectively improve arterial compliance not secondary to blood pressure lowering. Other beneficial actions of $\mathrm{C} 21$ include neuroprotection and improved glucose metabolism. One animal experiment reported that C21 could prevent vascular dementia. ${ }^{50}$ If these intervention effects can also be repeated in humans and with a positive tolerance profile, the drug could be used for vascular protection in combination with more conventional antihypertensive and lipid-lowering drugs. So far, the safety profile seems to be beneficial in the animal experiments, even at supranormal dosages of C21.

Another way to treatarterial stiffness is to try the effect of transcutaneous electrical nerve stimulation (TENS). This physical intervention applied on the skin has been evaluated in a randomised, controlled study in 24 healthy young adult and middle-aged men using the augmentation index (AIX) and haemodynamic measures. The results indicated that an acute application of ganglion TENS attenuated arterial stiffness in the younger adults as well as haemodynamic measures in the middleaged group. This method, the authors say, ${ }^{51}$ could emerge as effective therapy for the management of arterial blood pressure, but it must be proved in larger studies that are longer duration.

There have been high hopes for dietary modification of vascular ageing by use, for example, of resveratrol, an extract from red wine flavonoids. In a 6-month randomised, controlled trial, 56 non-obese (body mass index [BMI] 21-30 kg/m²) men and women, aged 38-55, were assigned to a dietary supplement (SUP) group or control (CON) group, with a 6-month follow-up.52 The SUP group took 10 dietary supplements each day (100 mg of resveratrol, a complex of $800 \mathrm{mg}$ each of green, black and white tea extracts; $250 \mathrm{mg}$ of pomegranate extract; $650 \mathrm{mg}$ of quercetin; $500 \mathrm{mg}$ of acetyl-I-carnitine; $600 \mathrm{mg}$ of lipoic acid; $900 \mathrm{mg}$ of curcumin; $1 \mathrm{~g}$ of sesamin; $1.7 \mathrm{~g}$ of cinnamon bark extract; and $1.0 \mathrm{~g}$ fish oil). Both the SUP and CON groups also took a daily multivitamin/mineral supplement. However, a total of 24 weeks' daily supplementation by these dietary supplements did not affect arterial stiffness or endothelial function in nonobese individuals. The authors thus concluded that supplementation with a combination of popular dietary supplements had no cardiovascular or metabolic effects in non-obese relatively healthy individuals. ${ }^{52}$

\section{Summary}

The description of EVA as a new model for research and early cardiovascular prevention has sparked interest among many physicians and brings a new perspective after the rise and fall of the metabolic syndrome. We need to better understand the double process of arteriosclerosis and atherosclerosis leading to CVD manifestations. Current medical and surgical therapies will be expanded in the future for better control of these pathological processes and even for the control of arterial ageing. New interventions are needed to address the role of glycaemia and AGE products for worsening EVA, as well as methods to counteract this detrimental influence on the arterial wall in all its layers. Better control of arterial stiffness and central haemodynamics ${ }^{53}$ could eventually translate into improved prognosis and reduced risk of CVD events. If C21 is safe and effective in humans, this could bring a new tool for vascular protection in combination with more traditional drugs for control of hypertension and hyperlipidaemia, as well as hyperglycaemia. Time will tell.
1. Anderson KM, Wilson PW, Odell PM, et al., An updated coronary risk profile: A statement for health professionals, Circulation, 1991;83:356-62.

2. Thomsen TF, Davidsen $\mathrm{M}$, Ibsen $\mathrm{H}$, et al., A new method for CHD prediction and prevention based on regional risk scores and randomized clinical trials; PRECARD and the copenhagen Risk Score, I Cardiovasc Risk, 2001;8:291-7.

3. Thomsen TF, McGee D, Davidsen M, et al., A cross-validation of risk-scores for coronary heart disease mortality based on data from the Glostrup Population Studies and Framingham Heart Study, Int J Epidemiol, 2002;31:817-22.

4. Eichler K, Puhan MA, Steurer J, et al., Prediction of first coronary events with the Framingham score: a systematic review, Am Heart J, 2007:153:722-31, 731.e1-8.

5. HalcoX JP, Tubach F, Sazova O, et al., on behalf of the EURIKA steering committee, Reclassification of European patients' cardiovascular risk using the updated Systematic Coronary Risk Evaluation algorithm, Eur J Prev Cardiology, 2015;22:200-2

6. Chamnan P, Simmons RK, Sharp SJ, et al., Cardiovascular risk assessment scores for people with diabetes: a systematic review, Diabetologia, 2009;52:2001-14.

7ethelius B, Eliasson B, Eeg-Olofsson K, et al., NDR, A new model for 5-year risk of cardiovascular disease in type 2 diabetes, from the Swedish National Diabetes Register (NDR) Diabetes Res Clin Pract, 2011;93:276-84.
8. Paoletti R, Bolego C, Poli A, et al., Metabolic syndrome, inflammation and atherosclerosis, Vasc Health Risk Manag, 2006;2:145-52.

9. Burgess S, Butterworth A, Malarstig A, et al., Use of Mendelian randomisation to assess potential benefit of clinical intervention, BMJ, 2012;345:e7325.

10. Kahn R, Buse J, Ferrannini E, et al., The metabolic syndrome: time for a critical appraisal; joint statement from the American Diabetes Association and the European Association for the Study of Diabetes, Diabetologia, 2005;48:1684-99.

11. Najjar SS, Scuteri A, Lakatta EG, Arterial aging: is it an immutable cardiovascular risk factor? Hypertension, 2005:46:454-62.

12. Hansson GK. Inflammation, atherosclerosis, and coronary artery disease, N Engl J Med, 2005;352:1685-95.

13. Gluckman PD, Hanson MA, Cooper C, et al., Effect of in utero and early-life conditions on adult health and disease N Engl J Med, 2008;359:61-73.

14. Nilsson PM, Boutouyrie P, Laurent S, Vascular aging: a tale of EVA and ADAM in cardiovascular risk assessment and prevention, Hypertension, 2009;54:3-10.

15. Nilsson PM, Boutouyrie P, Cunha P, et al., Early vascular ageing in translation: from laboratory investigations to clinical applications in cardiovascular prevention, J Hypertens, 2013:8:1517-26.
16. Scuteri A, Nilsson PM, Tzourio C, et al., Microvascular brain damage with aging and hypertension: pathophysiological consideration and clinical implications, J Hypertens, 2011;29:1469-77

17. Reference Values for Arterial Stiffness Collaboration, Determinants of pulse wave velocity in healthy people and in the presence of cardiovascular risk factors: establishing normal and reference values, Eur Heart 2010;31:2338-50.

18. Gottsäter M, Östling G, Persson M, et al., Non-hemodynamic predictors of arterial stiffness after 17 years of follow-up: The Malmö Diet and Cancer Study, J Hypertens, 2015 [Epub ahead of print].

19. Nilsson ED, Elmståhl S, Minthon L, et al., Nonlinear association between pulse wave velocity and cognitive function: population-based study, J Hypertens, 2014:32:2152-7.

20. Tragante V, Barnes MR, Ganesh SK, et al., Gene-centric metaanalysis in 87,736 individuals of European ancestry identifies multiple blood-pressure-related loci, Am J Hum Genet, 2014;94:349-60

21. Tarasov KV, Sanna S, Scuteri A, et al., COL4A1 is associated with arterial stiffness by genome-wide association scan, Circ Cardiovasc Genet, 2009;2:151-8.

22. Logan JG, Engler MB, Kim H, Genetic determinants of arterial stiffness, I Cardiovasc Trans/ Res, 2015;8:23-43. 
23. Denil SL, Rietzschel ER, De Buyzere ML, et al., Asklepios Investigators, On cross-sectional associations of leukocyte telomere length with cardiac systolic, diastolic and vascular function: the Asklepios study, PLOS One, 2014;9:e115071.

24. Ben-Shlomo Y, Spears M, Boustred C, et al., Aortic pulse wave velocity improves cardiovascular event prediction: an individual participant meta-analysis of prospective observational data from 17,635 subjects, J Am Coll Cardiol ، 2014;63:636-46.

25. Laurent S, Cockcroft J, Van Bortel L, et al., Expert consensus document on arterial stiffness: methodological issues and clinical applications, Eur Heart J, 2006;27:2588-605.

26. Van Bortel LM, Laurent S, Boutouyrie P, et al., Artery Society; European Society of Hypertension Working Group on Vascula Structure and Function; European Network for Non-invasive Investigation of Large Arteries, Expert consensus document on the measurement of aortic stiffness in daily practice using carotid-femoral pulse wave velocity, J Hypertens, 2012;30:445-8.

27. O'Rourke MF, Safar ME, Dzau V, The Cardiovascular Continuum extended: aging effects on the aorta and microvasculature, Vasc Med, 2010;15:461-8.

28. Cecelja M, Chowienczyk P, Dissociation of aortic pulse wave velocity with risk factors for cardiovascular disease other than hypertension: a systematic review, Hypertension. 2009;54:1328-36.

29. Al Ghatrif M, Strait JB, Morrell CH, et al., Longitudinal trajectories of arterial stiffness and the role of blood pressure: the Baltimore Longitudinal Study of Aging, Hypertension, 2013;62:934-41.

30. Kaess BM, Rong J, Larson MG, et al., Aortic stiffness, blood pressure progression, and incident hypertension, JAMA 2012;308:875-81.

31. Stehouwer $\mathrm{CD}$, Henry RM, Ferreira I, Arterial stiffness in diabetes and the metabolic syndrome: a pathway to cardiovascular disease, Diabetologia, 2008;51:527-39.

32. Prenner SB, Chirinos JA, Arterial stiffness in diabetes mellitus,
Atherosclerosis, 2015;238:370-9

33. Briet M, Pierre B, Laurent S, et al., Arterial stiffness and pulse pressure in CKD and ESRD, Kidney Int, 2012;82:388-400.

34. Johansen NB, Vistisen D, Brunner EJ, et al., Determinants of aortic stiffness: 16-year follow-up of the Whitehall II study, PLoS One, 2012:7:e37165.

35. Nilsson PM, Nilsson JA, Berglund G, Family burden of cardiovascular mortality: risk implications for offspring in a national register linkage study based upon the Malmö Preventive Project, J Intern Med, 2004:255:229-35.

36. Schloss PD, Iverson KD, Petrosino JF, et al., The dynamics of a family's gut microbiota reveal variations on a theme, Microbiome, 2014:2:25

37. Ettinger $R$, MacDonald $K$, Reid $G$, et al., The influence of the human microbiome and probiotics on cardiovascular health Gut Microbes, 2014;5:719-28.

38. Ong KT, Delerme $\mathrm{S}$, Pannier $\mathrm{B}$, et al., investigators, Aortic stiffness is reduced beyond blood pressure lowering by short-term and long-term antihypertensive treatment: a meta-analysis of individual data in 294 patients, J Hypertens, 2011;29:1034-42

39. Laurent S, Mousseaux E, Boutouyrie P, Arterial stiffness as an imaging biomarker: are all pathways equal? Hypertension, 2013;62:10-2.

40. Bonadei I Vizzardi E, D'Aloia A et al . Role of aliskiren on arterial stiffness and endothelial function in patients with primary hypertension, J Clin Hypertens (Greenwich), with primary hyper

41. Parving HH, Brenner BM, MCMurray JJ, et al., ALTITUDE nvestigators, Cardiorenal end points in a trial of aliskiren for type 2 diabetes, N Eng/ J Med, 2012;367:2204-13.

2. Laurent S, Schlaich M, Esler M, New drugs, procedures, and devices for hypertension, Lancet, 2012;380:591-600.

43. Ndip A, Wilkinson FL, Jude EB, et al., RANKL-OPG and RAGE modulation in vascular calcification and diabetes: novel targets for therapy, Diabetologia, 2014;57:2251-60.

44. Lanzer P, Boehm M, Sorribas V, et al., Medial vascular calcification revisited: review and perspectives, Eur Heart J, 2014;35:1515-25.

45. Gylling $\mathrm{H}$, Halonen J, Lindholm $\mathrm{H}$, et al., The effects of plant stanol ester consumption on arterial stiffness and endothelia function in adults: a randomised controlled clinical trial, BMC Cardiovasc Disord, 2013:13:50.

46. Lynn A, Mathew S, Moore CT, et al., Effect of a tart cherry juice supplement on arterial stiffness and inflammation in juice suppleme Hum Nutr, 2014:69:122-7.

47. Rydén L, Grant PJ, Anker SD, et al., ESC Guidelines on Rydén L, Grant PJ, Anker SD, et al., ESC Guidelines on
diabetes, pre-diabetes, and cardiovascular diseases developed in collaboration with the EASD: the Task Force on diabetes, pre-diabetes, and cardiovascular diseases of the European Society of Cardiology (ESC) and developed in collaboration with the European Association for the Study of Diabetes (EASD), Eur Heart J, 2013;34:3035-87.

48. Steckelings UM, Paulis L, Namsolleck P, et al., AT2 receptor agonists: hypertension and beyond, Curr Opin Nephrol Hypertens, 2012;21:142-6.

49. Paulis L, Becker ST, Lucht K, et al., Direct angiotensin II type 2 receptor stimulation in $\mathrm{N} \omega$-nitro-L-arginine-methyl esterinduced hypertension: the effect on pulse wave velocity and aortic remodeling, Hypertension, 2012;59:485-92.

50. Iwanami J, Mogi M, Tsukuda K, et al., Direct angiotensin II type 2 receptor stimulation by compound 21 prevents vascular 2 receptor stimulation by compound 21 prevents vascular

51. da Silva ML, Chiappa GR, da Silva VM, et al., Effect of transcutaneous electrical nerve stimulation on peripheral to central blood pressure ratio in healthy subjects, Cli Physiol Funct Imaging, 2015 [Epub ahead of print].

52. Soare A, Weiss EP, Holloszy JO, et al., Multiple dietary supplements do not affect metabolic and cardio-vascular health, Aging (Albany NY), 2014;6:149-57.

53. Asmar R, Effects of treatment on arterial stiffness and central blood pressure-points to consider, I Clin Hypertens (Greenwich), 2015;17:105-6. 